\title{
RESULTS FROM LHCF EXPERIMENT
}

\section{Alessia Tricomi*, on Behalf of the LHCF Collaboration}

\author{
University of Catania and INFN Catania, Italy \\ * corresponding author: alessia.tricomi@ct.infn.it
}

\begin{abstract}
The LHCf experiment took data in the 2009 and $2010 \mathrm{p}-\mathrm{p}$ collisions at LHC at $\sqrt{s}=0.9 \mathrm{TeV}$ and $\sqrt{s}=7 \mathrm{TeV}$. This paper reports on the most up-to-date results on the inclusive photon spectra and the $\pi^{0}$ spectra measured by LHCf. These spectra are compared with the model expectations, and the impact on high energy cosmic ray (HECR) Physics is discussed. In addition, we discuss the perspectives for future analyses as well as the program for the next data taking period, in particular the foreseen data taking in $\mathrm{p}-\mathrm{Pb}$ collisions.
\end{abstract}

KeYWORDs: LHC, hadron interaction, cosmic rays, Monte Carlo models.

\section{INTRODUCTION}

Dedicated extensive air shower experiments have been taking data for many years, and have contributed considerably to our understanding of High and Ultra High Energy Cosmic (UHECR) Ray Physics. Recently, in particular, the Pierre Auger Collaboration [1] and the Telescope Array Collaboration [2, thanks to the excellent performance of their hybrid detector arrays, have been providing new exciting observations of UHECRs. Although these recent results have brought a deeper insight into the properties of primary cosmic rays, there remain systematic uncertainties due to our poor knowledge of the nuclear interactions in the earth's atmosphere [3. A calibration of the energy scale in the range accessible at LHC, $10^{15} \div 10^{17} \mathrm{eV}$, provides crucial inputs for a better interpretation of primary cosmic ray properties, in the region between the "knee" and the GZK cut-off. The LHCf experiment is designed to measure the energy spectra and the transverse momentum of neutral particles in a very high pseudo-rapidity region $(\eta>8.4)$ thus providing precise data for testing and calibrating the hadronic interaction models used in Monte Carlo (MC) simulation of extensive air showers.

\section{The LHCF Detector}

The LHCf experiment comprises two independent position sensitive electromagnetic calorimeters, located on both sides of the ATLAS experiment, $140 \mathrm{~m}$ away from the LHC-IP1 interaction point, inside the zerodegree neutral absorber (Target Neutral Absorber, TAN). Charged particles from the IP are swept away by the inner beam separation dipole before reaching the TAN, so that only photons mainly from $\pi^{0}$ decays and neutral hadrons reach the LHCf calorimeters.

Each calorimeter (ARM1 and ARM2) has a double tower structure, with the smaller tower located at a zero degree collision angle, approximately covering the region with pseudo-rapidity $\eta>10$ and the larger tower, approximately covering the region with $8.4<\eta<10$. Four $X-Y$ layers of position sensitive detectors (scintillating fibers in ARM1, silicon microstrip detectors in ARM2) provide measurements of the transverse profile of the showers. The two tower structure allows us to reconstruct the $\pi^{0}$ decaying in two $\gamma$, hitting the two towers separately, hence providing very precise absolute energy calibration of the detectors. In the range $E>100 \mathrm{GeV}$, the LHCf detectors have energy and position resolutions for electromagnetic showers better than $5 \%$ and $200 \mu \mathrm{m}$, respectively. A detailed description of the LHCf experiment can be found in Ref. 4 .

\section{The Single Photon EnERGY SPECTRA}

The LHCf Collaboration has measured the single photon energy spectrum at $7 \mathrm{TeV}$ [5] and, more recently, at $900 \mathrm{GeV} \mathrm{p}-\mathrm{p}$ collisions [6]. To minimize the backgrounds and hence reduce the systematic uncertainties of the measurements, for both analyses only a subset of the collected data corresponding to clean and low luminosity fill, has been analysed. For the $7 \mathrm{TeV}$ analysis, the analysed data correspond to integrated luminosity of $0.68 \mathrm{nb}^{-1}$ and $0.52 \mathrm{nb}^{-1}$ for the ARM1 and ARM2 detectors, respectively, while for the $900 \mathrm{GeV}$ analysis they correspond to integrated luminosity of $0.30 \mathrm{nb}^{-1}$.

The main steps in the analysis work-flow are almost identical for the two analyses and are summarised in the following.

The energy of photons is reconstructed from the signal released by the shower particles in the scintillators, after applying corrections for the non-uniformity of light collection and for particles leaking in and out of the edges of the calorimeter towers. In order to correct for these last two effects, which are rather important due to the limited transverse size of the two calorimetric towers, we use the transverse impact position of the showers provided by the position sensitive detectors. 



FiguRE 1. Single photon energy spectra measured by LHCf (black dots) in $\eta>10.94$ (left) and $8.81<\eta<8.99$ (right) bin, respectively, for $7 \mathrm{TeV}$ (top panel) and $\eta>10.15$ (left) and $8.77<\eta<9.46$ (right) bin, respectively, for $900 \mathrm{GeV}$ (bottom panel) p-p collisions. The ratio of MC results predictions for DPMJET III 3.04 (red), QGSJET II-03 (blue), SIBYLL 2.1 (green), EPOS 1.99 (magenta) and PYTHIA 8.145 (yellow) to experimental data is shown. The error bars and gray shaded areas in each plot indicate the statistical errors and the systematic errors, respectively. Figures from Ref. [5, 6].

Events produced by neutral hadrons are rejected using information about the longitudinal development of the showers, which is different for electromagnetic and hadronic particles. In addition, for the $7 \mathrm{TeV}$ analysis, thanks to the information provided by the position sensitive detectors, events with more than one shower inside the same tower (multi-hit) are rejected, while for the $900 \mathrm{GeV}$ analysis the number of multiparticle events is negligible, hence multi-hit rejection is not applied. In order to combine the spectra measured by ARM1 and ARM2, which have different geometrical configurations, in these analyses only events detected in a common pseudo-rapidity and azimuthal range are selected: $\eta>10.94$ and $8.81<\eta<8.99$, for the small and large towers, respectively, for $7 \mathrm{TeV}$ analysis and $\eta>10.15$ and $8.77<\eta<9.46$, for the small and large towers, respectively, for $900 \mathrm{GeV}$ analysis.

Figure 1 shows the single $\gamma$ spectra measured by LHCf in the two pseudo-rapidity regions for $7 \mathrm{TeV}$ and $900 \mathrm{GeV}$ p-p collisions, respectively, compared with results predicted by MC simulations using different models: DPMJET III-3.04 [7], QGSJET II-03 [8], SIBYLL 2.1 9], EPOS 1.9 [10] and PYTHIA 8.145 [11]. Statical errors and systematic uncertainties are also plotted. A careful study of systematic uncertainties has been made, and conservative estimates have been taken into account. Further details can be found in
Ref. 5, 6. As can be seen from Fig. 1, there is a clear discrepancy between the experimental results and the predictions of the models, in particular in the high energy region.

\section{Neutral Pion transverse MOMENTUM SPECTRA}

In addition to the measurement of the single photon spectra, the LHCf experiment has recently finalised the measurements of the transverse momentum spectra for different rapidity bins for $\pi^{0}$ produced in $7 \mathrm{TeV}$ $\mathrm{p}-\mathrm{p}$ collisions at LHC. The integrated luminosities corresponding to the data used in this analysis are $2.53 \mathrm{nb}^{-1}$ (Arm1) and $1.90 \mathrm{nb}^{-1}$ (Arm2) after the data taking live times were taken into account. The $\pi^{0}$ are reconstructed in LHCf identifying their decays in two photons. Events are selected requiring that the two photons enter different calorimeter towers; due to the geometrical acceptance of the detector, only photons from $\pi^{0}$ decays with an opening angle of $\theta<0.4 \mathrm{mrad}$ can be detected. The energy, $p_{\mathrm{T}}$ and rapidity of the $\pi^{0}$ are reconstructed through measurements of the photon energy and the incident position in each calorimeter. In order to ensure good event reconstruction efficiency and geometrical acceptance, the range of the $\pi^{0}$ rapidity and transverse momentum 

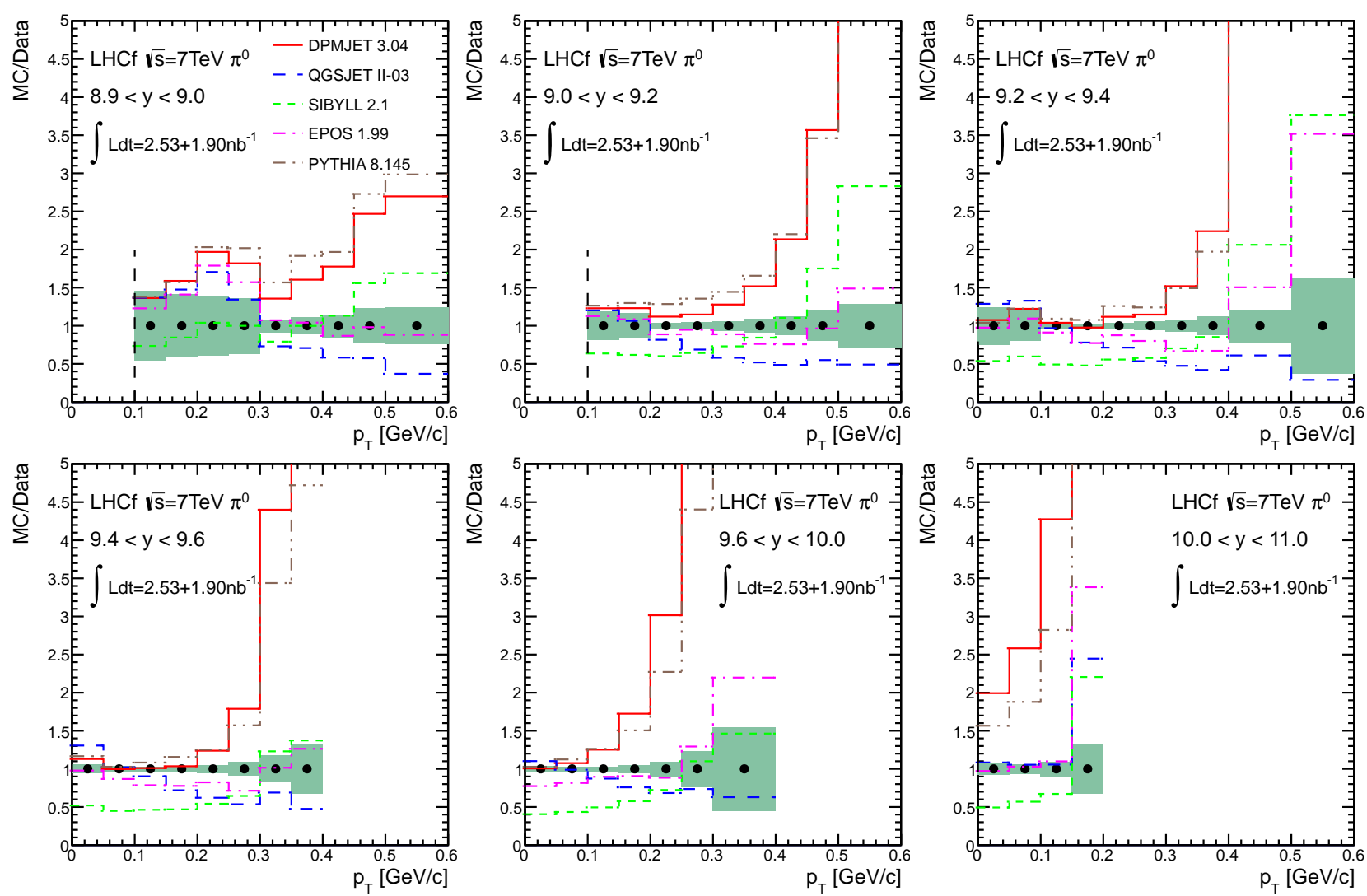

Figure 2. Ratio of the combined ARM1 and ARM2 $p_{\mathrm{T}}$ spectra to the $p_{\mathrm{T}}$ spectra predicted by various hadronic interaction models. Shaded areas indicate the range of total uncertainties of the combined spectra. Figure from Ref. [12].

are limited to $8.9<y<11.0$ and $p_{\mathrm{T}}<0.6 \mathrm{GeV} / \mathrm{c}$, respectively. Figure 2 shows the ratios of the $p_{\mathrm{T}}$ spectra predicted by DPMJET 3.04 (solid, red), QGSJET II03 (dashed, blue), SIBYLL 2.1 (dotted, green), EPOS 1.99 (dashed dotted, magenta), and PYTHIA 8.145 (default parameter set, dashed double-dotted, brown) to the combined ARM1 and ARM2 $p_{\mathrm{T}}$ spectra (black dots). Error bars have been taken from the statistical and systematic uncertainties. Among hadronic interaction models tested in this analysis, EPOS 1.99 shows the best overall agreement with the LHCf data, although it behaves softer than the data in the low $p_{\mathrm{T}}$ region, $p_{\mathrm{T}} \lesssim 0.4 \mathrm{GeV} / \mathrm{c}$ in $9.0<y<9.4$ and $p_{\mathrm{T}} \lesssim 0.3 \mathrm{GeV} / \mathrm{c}$ in $9.4<y<9.6$, and behaves harder in the large $p_{\mathrm{T}}$ region. DPMJET 3.04 and PYTHIA 8.145 show overall agreement with the LHCf data for $9.2<y<9.6$ and $p_{\mathrm{T}}<0.25 \mathrm{GeV} / \mathrm{c}$, while the expected $\pi^{0}$ productions rates by both models exceed the LHCf data for larger $p_{\mathrm{T}}$. Also SIBYLL 2.1 predicts harder pion spectra than the LHCf data, although the expected $\pi^{0}$ yield is generally small. Finally, QGSJET II-03 predicts $\pi^{0}$ spectra that are softer than the LHCf data and the other models.

\section{IMPACT OF LHCF RESULTS ON HECR PHYSICS}

The first LHCf results have attracted attention in the HECR community. As reported in the previous para- graphs, none of the models agree with the data in the whole energy range. Tuning of the models is therefore needed to describe the Physics of hadronic interactions at the $\mathrm{TeV}$ scale. In order to better understand the implications of this measurement for HECR Physics, collaboration has begun with several MC developers and theoreticians.

As an example, we have artificially modified the DPMJET III 3.04 model to produce a $\pi^{0}$ spectrum that differs from the original one by an amount approximately equal to the difference expected between the different models. Figure 3 shows the $\pi^{0}$ spectra at $E_{\text {lab }}=10^{17} \mathrm{eV}$ predicted by the original DPMJET III 3.04 model and the artificially modified spectra. A difference in the position of the shower maximum of the order of $30 \mathrm{~g} / \mathrm{cm}^{2}$ is observed.

Figure 4 shows, as an example, the most recent results from the Auger Collaboration [13] for the distribution of the $\left\langle X_{\max }\right\rangle$ variable as a function of the energy, which is the most commonly used method for inferring the composition of cosmic rays, compared with the model predictions for a proton-like (red lines) and an Iron-like (blue lines) cosmic ray component, respectively. The difference in the $\left\langle X_{\max }\right\rangle$ distributions for the two cases is of the order of $100 \mathrm{~g} / \mathrm{cm}^{2}$, hence a $30 \mathrm{~g} / \mathrm{cm}^{2}$ shift is a sizable difference, which may be reflected significantly in the interpretation of HECR data. 




FiguRE 3. $\pi^{0}$ spectra at $E_{\text {lab }}=10^{17} \mathrm{eV}$ for the original DPMJET III-3.04 model (red) and artificially modified models (green, blue, magenta), see text (top).

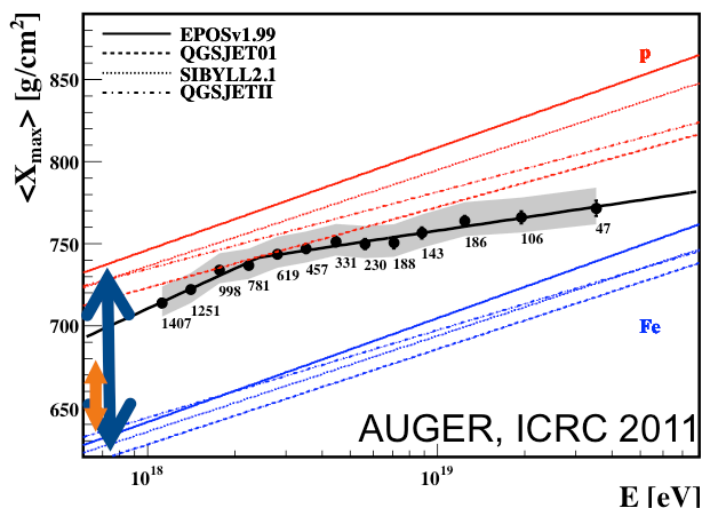

Figure 4. $\left\langle X_{\max }\right\rangle$ distribution as measured by Auger [13] (black points), compared with the model expectations for a light (red) or heavy (blue) cosmic ray composition. The yellow arrows correspond to the $30 \mathrm{~g} / \mathrm{cm}^{2}$ shift, obtained in Fig. 2

The importance of direct measurements of the $\gamma$ and $\pi^{0}$ spectra by LHCf turns on to be clear.

\section{Future ACtivities And Summary}

LHCf is planning to measure very forward particle emission in the LHC $\mathrm{p}-\mathrm{Pb}$ collisions foreseen at the beginning of 2013. The measurements are expected to constrain the nuclear effect in the forward particle emission relevant to the $\mathrm{CR}-\mathrm{Air}$ interaction, thus providing a further tool for model calibration [14. New analyses of data collected in the 2009-2010 runs are also in progress, in particular the measurements of the neutral hadron spectra. In the meantime, the LHCf Collaboration is working on upgrading the detector to improve the radiation resistance in view of the $14 \mathrm{TeV}$ p-p run, currently foreseen in 2014.

The scintillating part of the detector will be replaced by GSO slabs, thus enabling LHCf to sustain the radiation level foreseen in the $14 \mathrm{TeV}$ run [15]. Additional improvements in the front-end electronics of the silicon position sensitive layers of ARM2 de- tectors, as well as an optimization of the layout to improve the stand-alone silicon energy resolution, are also ongoing.

\section{REFERENCES}

[1] P. Abreu, et al. [The Pierre Auger Collaboration], arXiv:1107.4809 [astro-ph.HE]

[2] Y. Tsunesada [for the Telescope Array Collaboration], arXiv:1111.2507 [astro-ph.HE]

[3] A. Chiavassa, this Workshop

[4] O. Adriani et al., JINST 3 (2008) S08006

[5] O. Adriani et al., Phys. Lett. B703 (2011) 128

[6] O. Adriani et al., accepted in Phys. Lett. B, arXiv:1207.7183 [hep-ex]

[7] F.W. Bopp et al., Phys. Rev. C77 (2008) 014904

[8] S. Ostapchenko, Phys. Rev. D83 (2011) 014108

[9] E.-J. Ahn et al., Phys. Rev. D80 (2009) 094003

[10] K. Werner et al., Nucl.Phys.Proc.Suppl. 175-176 (2008) 81

[11] T. Sjöstand, et al., Comput. Phys. Comm. 178 (2008) 852

[12] O. Adriani et al., arXiv:1205.4578 [hep-ex]

[13] J.Bellido for the Auger Collaboration, in Proceedings of $32^{\text {st }}$ ICRC, Beijing, (2011)

[14] O. Adriani et al., CERN-LHCC-2011-015. LHCC-I-021 (2011)

[15] K. Kawade et al., JINST 6 (2011) T09004

\section{Discussion}

Laurence Jones - Does LHCf also have inclusive neutron spectra?

Alessia Tricomi - Currently the measurement of neutron spectra is our highest priority for future analysis. We have already started to work on that and we plan to be able to publish our results at the beginning of next year. It is a rather important measurement both for the implications in HECR Physics as well as for probing QCD Physics in the high-energy forward region. Although our detector is mainly designed to detect E.M. showers due to the limited interaction length $\left(\approx 1.6 \lambda_{\mathrm{i}}\right)$ resulting in an energy resolution of $\sim 30 \%$ for hadronic showers w.r.t. $\lesssim 3 \%$ for EM showers, simulation studies performed so far show that LHCf is pretty well able to disentangle between different hadronic models also in the neutron spectra due to the larger discrepancies present in the Monte Carlo models.

Aurelio Grillo - In which direction does $\left\langle X_{\max }\right\rangle$ move?

Alessia Tricomi - In the toy model that we have used, we have artificially modified DPMJET III 3.04 by softening the spectrum by an amount approximately equal to the difference expected between the different models. This modification of the spectrum reflects in a shift of the position of the shower maximum of the order of $30 \mathrm{~g} / \mathrm{cm}^{2}$ less than in the original model, moving from $\left\langle X_{\max }\right\rangle=718 \mathrm{~g} / \mathrm{cm}^{2}$ in the original DPMJET III 3.04 to $\left\langle X_{\max }\right\rangle=689 \mathrm{~g} / \mathrm{cm}^{2}$ in the modified one. Let me point out however that this was just an artificial modification, not a full recalibration of the model. 